Ark. Mat., 32 (1994), 13-32

(C) 1994 by Institut Mittag-Leffler. All rights reserved

\title{
Discrete spectrum of the perturbed Dirac operator
}

\author{
Mikhail Sh. Birman and Ari Laptev
}

\begin{abstract}
In this paper we study the asymptotics of the discrete spectrum in the gap $(-1,1)$ of the perturbed Dirac operator $\mathcal{D}(\alpha)=\mathcal{D}_{0}-\alpha V 1$ acting in $L_{2}\left(\mathbf{R}^{3} ; \mathbf{C}^{4}\right)$ with large coupling constant $\alpha$. In particular some "non-standard" asymptotic formulae are obtained.
\end{abstract}

\section{§1. Introduction}

§1.1. The Dirac operator with a decreasing electric potential gives a non-trivial example of the discrete spectrum in a gap of the continuous one. Let $\boldsymbol{\gamma}=\left(\gamma_{1}, \gamma_{2}, \gamma_{3}\right)$ and $\gamma_{0}$ be $(4 \times 4)$ Dirac matrices; 1 be the unit matrix. The Dirac matrices satisfy the equations

$$
\gamma_{j} \gamma_{k}+\gamma_{k} \gamma_{j}=2 \delta_{j k} 1, \quad j, k=0,1,2,3 .
$$

Let us consider the "free" Dirac operator in $L_{2}\left(\mathbf{R}^{3} ; \mathbf{C}^{4}\right)$

$$
\begin{gathered}
\mathcal{D}_{0}=\boldsymbol{\gamma} \cdot \boldsymbol{D}+\gamma_{0}, \\
\boldsymbol{D}=-i\left(\frac{\partial}{\partial x_{1}}, \frac{\partial}{\partial x_{2}}, \frac{\partial}{\partial x_{3}}\right), \quad \boldsymbol{\gamma} \cdot \boldsymbol{D}=-i \sum_{j=1}^{3} \gamma_{j} \frac{\partial}{\partial x_{j}},
\end{gathered}
$$

and its perturbation by a potential

$$
\begin{gathered}
\mathcal{D}(\alpha)=\mathcal{D}_{0}-\alpha V 1, \quad \alpha>0, \\
V \in L_{3}\left(\mathbf{R}^{3}\right), \quad V(x) \geq 0 .
\end{gathered}
$$

The spectrum of the operator $\mathcal{D}_{0}$ is (absolutely) continuous and covers the complement of the interval (gap) $\Lambda=(-1,1)$. The continuous spectrum of the operator 
$\mathcal{D}(\alpha)$ coincides with the continuous spectrum of $\mathcal{D}_{0}$. Besides, the operator $\mathcal{D}(\alpha)$ has a discrete spectrum in the gap $\Lambda$. The eigenvalues of $\mathcal{D}(\alpha)$ are monotonically moving to the left, when $\alpha$ is increasing.

Let us denote by $N(\alpha, \lambda)$ the number of eigenvalues of the operator $\mathcal{D}(t)$ passing the point $\lambda,|\lambda| \leq 1$, when the coupling constant $t$ is increasing from 0 to the value $t=\alpha$. We study the asymptotic behaviour of $N(\alpha, \lambda)$ when $\alpha \rightarrow \infty$. In the case $\lambda= \pm 1$ we need some additional assumptions on $V$. In particular, these assumptions guarantee $N(\alpha, 1)$ to be finite for all $\alpha>0$.

The starting point of our paper is the following result of $[\mathrm{K}]$.

Proposition $1.1([\mathbf{K}])$. Let us assume that, as well as (1.3), we have

$$
V \in L_{3 / 2}\left(\mathbf{R}^{3}\right)
$$

Then the following asymptotic formula holds*

$$
\lim _{\alpha \rightarrow \infty} \alpha^{-3} N(\alpha, \pm 1)=\frac{1}{3 \pi^{2}} \int V^{3} d x .
$$

In this paper we claim the following statements in addition to the result of Klaus.

(a) The asymptotic formula (1.5) holds for $N(\alpha, \lambda),|\lambda|<1$, whenever we only have the condition (1.3).

(b) The asymptotic formula (1.5) survives under some weaker (compare with (1.4)) additional restrictions on $V$.

(c) There are potentials satisfying (1.3), such that $N(\alpha, 1)$ has an asymptotics of the order $\alpha^{q}, q>3$.

(d) There are potentials, satisfying (1.3), such that $N(\alpha, 1) \sim c \alpha^{3}$, but the coefficient $c>J$, where

$$
J:=\frac{1}{3 \pi^{2}} \int V^{3} d x
$$

$\S 1.2$. Let us clarify the previous statements. We begin with the necessary notations.

Let $T$ be a compact operator in a separable Hilbert space, and $s_{k}(T)$ be its singular numbers, i.e.; the decreasing sequence of the eigenvalues of the operator $\left(T^{*} T\right)^{1 / 2}$, enumerated with their multiplicity. Let us set

$$
n(s, T)=\operatorname{card}\left\{k \in \mathbf{N}: s_{k}(T)>s\right\}, \quad s>0 .
$$

Besides, if $T=T^{*}$ we set $2 T_{ \pm}=|T| \pm T$, and

$$
n_{ \pm}(s, T):=n\left(s, T_{ \pm}\right), \quad s>0 .
$$

\footnotetext{
* The coefficient in $[\mathrm{K}]$ is incorrect and this mistake is repeated in [T].
} 
Obviously

$$
n(s, T)=n_{+}(s, T)+n_{-}(s, T), \quad T=T^{*} .
$$

Throughout the paper we use the notation

$$
W(x)=(V(x))^{1 / 2} .
$$

Let us consider the operator

$$
X(\lambda)=W\left(\mathcal{D}_{0}-\lambda I\right)^{-1} W, \quad|\lambda| \leq 1 .
$$

If this operator is compact, then the well known abstract method gives

$$
N(\alpha, \lambda)=n_{+}(s, X(\lambda)), \quad \alpha s=1,|\lambda| \leq 1 .
$$

Using the relations (1.1) we represent the operator (1.6) as follows

$$
\begin{gathered}
X(\lambda)=Y(\lambda)+Z(\lambda), \quad|\lambda| \leq 1, \\
Y(\lambda)=W\left(\gamma \cdot D(-\Delta+\sigma I)^{-1}\right) W, \quad \sigma=1-\lambda^{2}, \\
Z(\lambda)=W\left(\gamma_{0}+\lambda \mathbf{1}\right)(-\Delta+\sigma I)^{-1} W, \quad \sigma=1-\lambda^{2} .
\end{gathered}
$$

In particular, for the edges of the gap (i.e., when $\lambda= \pm 1$ )

$$
Y:=Y( \pm 1)=W\left(\boldsymbol{\gamma} \cdot \boldsymbol{D}|\boldsymbol{D}|^{-2}\right) W,
$$

$$
Z( \pm 1)=W\left(\gamma_{0} \pm \mathbf{1}\right)|\boldsymbol{D}|^{-2} W
$$

The operators $(1.9),(1.10)$ are pseudodifferential operators of order $(-1)$ and $(-2)$ respectively. The symbols of the operators (1.11), (1.12) are homogeneous. Besides,

$$
\pm Z( \pm 1) \geq 0 .
$$

Let us notice that the representation (1.8) - (1.10) together with (1.7) was used in $[\mathrm{K}]$.

The general results of the paper [BS2], about the Weyl asymptotics for the spectrum of pseudodifferential operators of negative order, imply (see §2.2) the following Proposition. 
Proposition 1.2. Under condition (1.3) we have the asymptotics

$$
\lim _{s \rightarrow 0} s^{3} n_{+}(s, Y(\lambda))=J, \quad|\lambda| \leq 1 .
$$

According to (1.7), (1.8) the asymptotics (1.14) is equivalent to

$$
\lim _{\alpha \rightarrow \infty} \alpha^{-3} N(\alpha, \lambda)=J,
$$

whenever

$$
n(s, Z(\lambda))=o\left(s^{3}\right) .
$$

We shall see below that if $|\lambda|<1$, then (1.16) is provided only under condition (1.3). On the contrary, if $\lambda= \pm 1$, then (1.16) cannot be obtained without some restrictions on $V$. One of the simplest restrictions of this type is the condition (1.4), which leads to the estimate

$$
n(s, Z( \pm 1))=O\left(s^{-3 / 2}\right)
$$

(see the explanations in §2.2). However, (1.16) can be obtained under some weaker restrictions on $V$. On the other hand, there are conditions on $V$, such that (1.3) is fulfilled, but

$$
n(s, Z( \pm 1))=O\left(s^{-q}\right), \quad q \geq 3 .
$$

Then the asymptotics (1.5) does not hold for sure. The most delicate case is $q=3$ in (1.18). Then the contribution of both terms in the representation

$$
X( \pm 1)=Y+Z( \pm 1)
$$

have the same order. We are going to show that for a sufficiently large class of potentials these contributions lead to the summation of their respective asymptotic coefficients (see Theorems 3.7 and 3.8).

§1.3. Two clarifications should be added to the above statements. Firstly, the operator (1.2) needs to be correctly defined. Having only the condition (1.3), it is impossible to introduce this operator as the sum of two operators. However, we can define the sum of the two operators in (1.2) via the sum of their respective sesquilinear forms. This definition is normally used for semibounded operators. For non-semibounded operators the scheme of definition of operators via the sum of forms is given in Chapter 1 of the book [Y]. In our case it can be applied under 
condition (1.3), since the operator $W\left|\mathcal{D}_{0}\right|^{-1 / 2}$ is compact. The reader can find all the details in $[\mathrm{Y}]$.

The second clarification concerns the relation (1.7) and some of its analogies. These types of formulae are well known in the additive perturbation theory. However, their justifications need an accuracy when the perturbation is introduced via the sum of forms, particularly, in the case when $\lambda$ is an edge of the gap. In the semibounded case all the details can be found in [B1] and [B2]. Similar ideas lead also to (1.7) in our case, if one introduces the sum of sesquilinear forms according to $[\mathrm{Y}]$.

Acknowledgement. We both wish to express our gratitude to the Mittag-Leffler Institute where this research was carried out.

\section{§2. The preliminary information}

Our analysis is based on the well developed technique of investigation of the negative discrete spectrum of the Schrödinger operator. We list here the important facts, with their references.

$\S 2.1$. Let us begin with the notations for classes of operators and functional spaces. Let $\mathfrak{H}$ be a separable Hilbert space. The space of linear continuous operators in $\mathfrak{H}$ is denoted by $\mathcal{B}=\mathcal{B}(\mathfrak{H})$; the space of compact operators in $\mathfrak{H}$ is equal to $\mathfrak{S}_{\infty}$. Furthermore, let $\mathfrak{S}_{p}$ be the class of operators $T \in \mathfrak{S}_{\infty}$ whose quasinorm

$$
\|T\|_{\mathfrak{S}_{p}}=\left(\sum_{k} s_{k}^{p}(T)\right)^{1 / p}, \quad 0<p<\infty,
$$

is finite. An operator $T \in \mathfrak{S}_{\infty}$ is said to be from the class $\Sigma_{p}$ if the quasinorm

$$
\|T\|_{\Sigma_{p}}=\sup _{s>0}\left(s^{p} n(s, T)\right)^{1 / p}, \quad 0<p<\infty
$$

is finite. The space $\Sigma_{p}$ is a complete and a non-separable space. By $\Sigma_{p}^{0}$ we denote the separable subspace

$$
\Sigma_{p}^{0}=\left\{T \in \Sigma_{p}: n(s, T)=o\left(s^{-p}\right)\right\}, \quad 0<p<\infty .
$$

Let us point out that $\mathfrak{S}_{p} \subset \Sigma_{p}^{0}, p>0$.

If $T \in \Sigma_{p}$ we introduce the following functionals (see [BS3])

$$
\Delta_{p}(T)=\limsup _{s \rightarrow 0} s^{p} n(s, T), \quad \delta_{p}(T)=\liminf _{s \rightarrow 0} s^{p} n(s, T) .
$$


If $T=T^{*} \in \Sigma_{p}$, then we also define the functionals

$$
\Delta_{p}^{ \pm}(T):=\Delta_{p}\left(T_{ \pm}\right), \quad \delta_{p}^{ \pm}(T):=\delta_{p}\left(T_{ \pm}\right)
$$

All these six functionals (2.2) and (2.3) are correctly defined and continuous on the factor-space $\Sigma_{p} / \Sigma_{p}^{0}$. Moreover, they are continuous with respect to the quasinorm (2.1).

Let $f(x)>0$ now be a measurable function on $\mathbf{R}^{d}$, which defines the measure $\varrho_{f}(\omega)=\int_{\omega} f d x, \omega \subset \mathbf{R}^{d}$. Let us recall the definition of the Lorentz spaces with respect to the measure $\varrho_{f}$ (we shall omit $f$ in our notations when $f(x)=1$ ). For a measurable function $u: \mathbf{R}^{d} \rightarrow \mathbf{C}$ we set

$$
\mu_{u}(t)=\varrho_{f}\left\{x \in \mathbf{R}^{d}:|u(x)|>t\right\} .
$$

We say that $u$ is in the class $L_{p, r}\left(\mathbf{R}^{d}, f\right), 0<p<\infty, 0<r \leq \infty$, provided that the quasinorm \|\|$_{p, r}$ is finite, where

$$
\begin{aligned}
\|u\|_{p, r}^{p} & =\int_{0}^{\infty} t^{r-1} \mu_{u}^{r / p}(t) d t, \quad 0<r<\infty \\
\|u\|_{p, \infty}^{p} & =\sup _{t>0} t^{p} \mu_{u}(t) .
\end{aligned}
$$

The spaces $L_{p, r}$ are complete; the spaces $L_{p, \infty}$ are not separable. When $r=p$ we obtain the usual classes $L_{p}\left(\mathbf{R}^{d}, f\right)$. Finally,

$$
L_{p, \infty}^{0}\left(\mathbf{R}^{d}, f\right)=\left\{u \in L_{p, \infty}\left(\mathbf{R}^{d}, f\right): \mu_{u}(t)=o\left(t^{-p}\right), t \rightarrow 0, t \rightarrow \infty\right\} .
$$

§2.2. In what follows below, $\Phi$ is the operator of the Fourier transform in $L_{2}\left(\mathbf{R}^{d}\right)$. By $C$ (may be with indices) we denote different constants appearing in our estimates. For the operator of multiplication by a function we use the same symbol as for the function itself.

\section{Proposition 2.1.}

(a) Let $b \in L_{p}\left(\mathbf{R}^{d}\right), c \in L_{p, \infty}\left(\mathbf{R}^{d}\right), p>2$. Then $b \Phi^{*} c \in \Sigma_{p}$ and the following estimate holds

$$
\left\|b \Phi^{*} c\right\|_{\Sigma_{p}} \leq C(p, d)\|b\|_{L_{p}}\|c\|_{L_{p, \infty}} .
$$

(b) Under the additional condition

$$
\operatorname{mes}\left\{x \in \mathbf{R}^{d}:|c(x)|>t\right\}=o\left(t^{-p}\right), \quad t \rightarrow 0,
$$

we have

$$
b \Phi^{*} c \in \Sigma_{p}^{0}
$$


In essence Proposition 2.1 is due to Cwikel [C]. Concerning (2.4) see [BKS], [BS5]. In the future we use the notation

$$
Q_{\varrho}:=W|D|^{-\varrho / 2}, \quad 0<\varrho<2 d .
$$

It is clear that $Q_{\varrho}$ is equal to $W \Phi^{*} c$, where $c(\xi)=|\xi|^{-\varrho / 2}$. Therefore we have

Proposition 2.2. Let $W \in L_{p}\left(\mathbf{R}^{d}\right), p>2$. Then

$$
\left\|Q_{\varrho}\right\|_{\Sigma_{p}} \leq C(p, d)\|W\|_{L_{p}}, \quad \varrho=2 d / p .
$$

Let $a(\vartheta), \vartheta=\xi /|\xi|$, now be a Hermitian matrix-function. The operator

$$
K_{\varrho}(a)=Q_{\varrho} a Q_{\varrho}^{*}
$$

is a pseudodifferential operator with the Hermitian symbol

$$
G(x, \xi):=|W(x)|^{2} a(\vartheta)|\xi|^{-\varrho}, \quad \vartheta=\xi /|\xi| .
$$

Proposition 2.3. Let $W \in L_{2 \varkappa}\left(\mathbf{R}^{d}\right), \varkappa>1, \varrho=d / \varkappa$ and $a \in L_{\infty}\left(S^{d-1}\right)$. Then

(a) The following estimate holds for the operator (2.7)

$$
\left\|K_{\varrho}(a)\right\|_{\Sigma_{\varkappa}} \leq C(d, \varkappa)\|a\|_{L_{\infty}}\|W\|_{L_{2 \varkappa}}^{2} .
$$

(b) The following asymptotic formula holds

$$
\Delta_{\varkappa}^{( \pm)}\left(K_{\varrho}(a)\right)=\delta_{\varkappa}^{( \pm)}\left(K_{\varrho}(a)\right)=(2 \pi)^{-d} \iint n_{ \pm}(1, G(x, \xi)) d x d \xi .
$$

(c) The asymptotics (2.9) hold if the function a( $\vartheta)$ in (2.7) is substituted by the sum $a(\vartheta)+g(\xi)$, where the Hermitian matrix $g \in L_{\infty}\left(\mathbf{R}^{d}\right)$ and $g(\xi) \rightarrow 0$ when $|\xi| \rightarrow \infty$.

Notice that the estimate (2.8) directly follows from (2.6). The subsections (b) and (c) in Proposition 2.3 are very special cases of the results of the paper [BS2] (see Theorem 1 and Remark 5 on this theorem, and also Theorem 2 and Remark 3 on this theorem).

It is easy to see that the asymptotics (1.14) are a simple corollary of Proposition 2.3. If $\lambda= \pm 1$ then, for the proof of (1.14), it is enough to use subsection (b). When $|\lambda|<1$ we must also take into account subsection (c). The arguments in the paper $[\mathrm{K}]$ are more cumbersome; instead of referring to the result from [BS2], the problem was reduced to the results of the earlier paper [BS1] which needs the homogeneity of the symbols. Probably this explains why in [K] the asymptotics (1.14) 
was obtained only for $\lambda= \pm 1$. Proposition 1.1 also follows directly from Proposition 2.3. Indeed, under condition (1.4) the estimate (1.17) follows from (2.8) when $d=3, \varkappa=\frac{3}{2}$ and $\varrho=2$.

\$2.3. To avoid tedious provisos, we come back to $d=3$. Apart from the operator $\mathcal{D}(\alpha)$ we consider in $L_{2}\left(\mathbf{R}^{3}\right)$ the Schrödinger operator

$$
H=-\Delta-\alpha V \text {. }
$$

We denote by $N_{H}(\alpha, \sigma), \sigma \geq 0$, the number of eigenvalues of the operator $H$, lying to the left of the point $(-\sigma)$. Let us also consider the analogy of the operator (1.6):

$$
Z_{H}(\sigma)=W(-\Delta+\sigma I)^{-1} W, \quad \sigma \geq 0,
$$

and observe the relation

$$
N_{H}(\alpha, \sigma)=n\left(s, Z_{H}(\sigma)\right), \quad \alpha s=1, \sigma \geq \mathbf{0} .
$$

If $\sigma=0$ we use the shortened notation

$$
Z_{H}:=Z_{H}(0)=Q_{2} Q_{2}^{*}
$$

The matrix $\gamma_{0} \pm 1$ is unitarily equivalent to the matrix $\pm 2 \operatorname{diag}(1,1,0,0)$. This implies that

$$
\begin{aligned}
\Delta(Z( \pm 1)) & =2^{p+1} \Delta_{p}\left(Z_{H}\right), \\
\delta(Z( \pm 1)) & =2^{p+1} \delta_{p}\left(Z_{H}\right) .
\end{aligned}
$$

Assume now that (1.4) is satisfied. It follows from (2.6) that

$$
\left\|Q_{2}\right\|_{\Sigma_{3}} \leq C|| W \|_{L_{3}} .
$$

By (2.11) this is equivalent to the inequality

$$
N_{H}(\alpha, \sigma) \leq C \alpha^{3 / 2} \int V^{3 / 2} d x, \quad \sigma \geq 0 .
$$

The last estimate is well known (Rozenblum-Lieb-Cwikel estimate). It is accompanied by the asymptotic formula

$$
\lim _{\alpha \rightarrow \infty} \alpha^{-3 / 2} N_{H}(\alpha, \sigma)=\frac{1}{6 \pi^{2}} \int V^{3 / 2} d x, \quad \sigma \geq 0 .
$$

§2.4. Let us now discuss the estimates for $N_{H}(\alpha, 0)$ when the condition (1.4) is broken. In particular, we need some sufficient conditions which guarantee the inclusion

$$
Z_{H} \in \Sigma_{q}, \quad q>\frac{3}{2} .
$$

The appropriate material is borrowed from [BS4], §4. Here we write down the estimates assuming that the right hand sides are finite. We regard the function $\varphi$, such that

$$
\varphi(x)>0, \quad \varphi \in L_{3 / 2, \infty}\left(\mathbf{R}^{3}\right), \quad\|\varphi\|_{L_{3 / 2, \infty}} \leq 1,
$$

to be fixed. As $\varphi$ we can take, for example, the normalized function $|x|^{-2}$. 
Proposition 2.4. If $q \geq \frac{3}{2}$, then

$$
\begin{gathered}
\left\|Z_{H}\right\|_{\mathfrak{S}_{q}}^{q} \leq C(q) \int V^{q} \varphi^{3 / 2-q} d x \\
\left\|Z_{H}\right\|_{\Sigma_{q}}^{q} \leq C(q) \sup _{t>0}\left(t^{q} \int_{V>t \varphi} \varphi^{3 / 2} d x\right), \\
\Delta_{q}^{q}\left(Z_{H}\right) \leq C(q) \limsup _{t+t^{-1} \rightarrow \infty}\left(t^{q} \int_{V>t \varphi} \varphi^{3 / 2} d x\right) .
\end{gathered}
$$

Let us clarify that the finiteness of the right hand sides in (2.17) and (2.18) means that the function $V / \varphi$ belongs to the classes $L_{q}\left(\varphi^{3 / 2}\right)$ and $L_{q, \infty}\left(\varphi^{3 / 2}\right)$ respectively. The right hand sides in (2.18) and (2.19) are finite, for instance, if

$$
V=\varphi|\ln \varphi|^{-\tau}, \quad \tau q=1 .
$$

These results look even simpler for some specially chosen functions $\varphi$. For example, the following estimate is the particular case of the estimate (2.17)

$$
\left\|Z_{H}\right\|_{\mathscr{S}_{q}}^{q} \leq C(q) \int V^{q}|x|^{2 q-3} d x .
$$

Futhermore, let $\chi_{R}$ be the characteristic function of the ball $\{|x|<R\}$ and $\Psi \in$ $L_{q}\left(\mathbf{S}^{2}\right), \Psi(\theta) \geq 0, \theta=x /|x|$. Let us consider the potential

$$
V_{\tau}(x)=\left(1-\chi_{1}(x)\right)|x|^{-2}(\ln |x|)^{-\tau} \Psi(\theta), \quad \tau^{-1}=q>\frac{3}{2},
$$

for which the estimate (2.18) turns out to be (if $\varphi=|x|^{-2}$ ) equivalent to the inequality

$$
s^{q} n\left(s, Z_{H}\right) \leq C(q) \int_{\mathrm{S}^{2}} \Psi(\theta)^{q} d S(\theta), \quad q>\frac{3}{2} .
$$

The accuracy of the estimate (2.21) is verified by the asymptotic formula which is essentially not a Weyl type asymptotic formula. Let us consider in $L_{2}\left(\mathbf{S}^{2}\right)$ the operator with a parameter $s>0$

$$
-\Delta_{\theta}-s^{-\tau} \Psi(\theta),
$$

where $\Delta_{\theta}$ is the Laplace operator on the unit sphere. Let $\left\{\nu_{l}^{(\tau)}(s)\right\}$ be the sequence of the eigenvalues of the operator (2.22) and

$$
M_{\tau}:=\frac{1}{\pi} \sum_{l} \int_{0}^{\infty}\left(\nu_{l}^{(\tau)}(s)+\frac{1}{4}\right)_{-}^{1 / 2} d s
$$


Proposition 2.5. Let, in (2.10), $V=V_{\tau}$, where $V_{\tau}$ is the potential defined by (2.20). Then the following asymptotic formula holds

$$
\lim _{s \rightarrow 0} s^{q} n\left(s, Z_{H}\right)=M_{\tau}, \quad \tau q=1, q>\frac{3}{2}
$$

The asymptotic formula $(2.24)$ is obtained in [L]; the partial result with $\Psi=1$ was analyzed earlier in [BS4], §8.

$\S 2.5$. Let us give one more simple estimate for the operator $Z_{H}$, which has not been mentioned before.

Propositon 2.6. The following implication is valid

$$
V \in L_{3 / 2, q}\left(\mathbf{R}^{3}\right) \Rightarrow Z_{H} \in \mathfrak{S}_{q}, \quad \frac{3}{2}<q<\infty
$$

Proof. It is more convenient to consider the operator $Q_{2}$ (see (2.5) and (2.12)). For this operator we have the implications

$$
\begin{aligned}
& W \in L_{3, \infty}\left(\mathbf{R}^{3}\right) \quad \Rightarrow \quad Q_{2} \in \mathcal{B}, \\
& W \in L_{3}\left(\mathbf{R}^{3}\right) \quad \Rightarrow \quad Q_{2} \in \Sigma_{3}
\end{aligned}
$$

accompanied by their respective estimates. The relation (2.26) is contained in the results of the paper [BKS], and (2.27) coincide with (2.15). It now remains to apply the real interpolation. The coincidence of the left first (main) indices is not an obstacle, since the main indices on the right hand sides of implications (2.26) and (2.27) coincide with the second indices on the left. The corresponding theorem concerning reiteration (see [BL] Theorem 3.5.4) allows one to deduce directly from (2.26) and (2.27) the following relation

$$
W \in L_{3 / 2,2 q} \quad \Rightarrow \quad Q_{2} \in \mathfrak{S}_{2 q}
$$

which is equivalent to $(2.25)$.

$\S 2.6$. Finally, let us mention one special result about the operator $Z_{H}(\sigma)$, when $\sigma>0$. It is contained in the paper [BS4] (Corollary 4.1 when $l=1, d=3, q=3$ ). However, we give here its direct deduction. 
Proposition 2.7. Under condition (1.3) we have

$$
Z_{H}(\sigma) \in \mathfrak{S}_{3}, \quad \sigma>0
$$

Proof. Let us take advantage of the representation $Z_{H}(\sigma)=Q_{2}(\sigma) Q_{2}(\sigma)^{*}$, with $Q_{2}(\sigma)=W(-\Delta+\sigma I)^{-1 / 2}$. The following implications are valid

$$
\begin{aligned}
& W \in L_{\infty}\left(\mathbf{R}^{3}\right) \Rightarrow Q_{2}(\sigma) \in \mathcal{B}, \quad \sigma>0, \\
& W \in L_{3}\left(\mathbf{R}^{3}\right) \Rightarrow Q_{2}(\sigma) \in \Sigma_{3}, \quad \sigma \geq 0 .
\end{aligned}
$$

The first one is obvious, and the second one follows from (2.15). After interpolation we obtain

$$
W \in L_{6}\left(\mathbf{R}^{3}\right) \Rightarrow Q_{2}(\sigma) \in \mathfrak{S}_{6}, \quad \sigma>0
$$

which is equivalent to $(2.28)$.

\section{\$3 The main results}

We shall now formulate the main results of the paper. After the preparation which has been done in $\S 1$ and $\S 2$, these results almost do not need any proofs, and therefore we shall give the necessary explanations at once. The only exception is Theorem 3.7. We devote $\S 4$ to the proof of this theorem.

$\S 3.1$.

Theorem 3.1. Under conditions (1.3) and $|\lambda|<1$ the relation (1.15) holds.

Proof. From (2.29) with $\sigma=1-\lambda^{2}$ we immediately obtain that

$$
Z(\lambda) \in \mathfrak{S}_{3}\left(\subset \Sigma_{3}^{0}\right), \quad|\lambda|<1
$$

This implies (1.16) and, therefore, the matter is reduced to Proposition 1.2.

$\S 3.2$. The following concerns the edges of the gap $\lambda= \pm 1$. The next proposition is almost obvious, but it is convenient to single it out. 
Proposition 3.2. Let the condition (1.3) be fulfilled, and assume that for the operator (2.12) we have the inclusion

$$
Z_{H} \in \Sigma_{3}^{0}
$$

Then the asymptotic formula (1.5) holds.

Proof. The proof once again is reduced to Proposition 1.2, since (1.16) is the corollary of (3.1) and (2.13) when $\lambda= \pm 1$.

The informative conditions for the validity of (3.1) can be extracted from the assertions of $\S 2.4$ and $\S 2.5$. We deduce some of the results avoiding the most general statements. The estimate (2.17) obviously leads to

Theorem 3.3. Let the condition (1.3) be fulfilled. Let, for some $q$, the integral

$$
\int V^{q}|x|^{2 q-3} d x<\infty, \quad \frac{3}{2} \leq q \leq 3,
$$

converge or, more generally,

$$
\int V^{q} \varphi^{3 / 2-q} d x<\infty, \quad \frac{3}{2} \leq q \leq 3,
$$

with some function $\varphi$, satisfying (2.16). Then the asymptotic formula (1.5) is valid.

The extreme value $q=\frac{3}{2}$ in (3.2) and (3.3) corresponds to the condition (1.4). The sufficient conditions (3.2) or (3.3) do not absorb each other for different values of $q$. The next theorem contains a more direct generalization of the condition (1.4)*

Theorem 3.4. Let us assume that together with (1.3) we have

$$
V \in L_{3 / 2,3}\left(\mathbf{R}^{3}\right)
$$

Then the asymptotic formula (1.5) holds.

Proof. By (2.25) from (3.4) we have (3.1).

§3.3. Let us consider the case when the operator $Z( \pm 1)$ dominates in the sum (1.19). It is also convenient to begin with the simplest statement.

* It can be shown that Theorem 3.4 absorbs Theorem 3.3. The authors thank T. Weidl for verifying this fact. 
Proposition 3.5. Let the condition (1.3) be satisfied and there be $q>3$ such that we have the following inclusion for the operator (2.12)

$$
Z_{H} \in \Sigma_{q}, \quad q>3
$$

Then

$$
\begin{gathered}
\limsup _{\alpha \rightarrow \infty} \alpha^{-q} N(\alpha, 1)=2^{q+1} \limsup _{\alpha \rightarrow \infty} \alpha^{-q} N_{H}(\alpha, 0), \\
\liminf _{\alpha \rightarrow \infty} \alpha^{-q} N(\alpha, 1)=2^{q+1} \liminf _{\alpha \rightarrow \infty} \alpha^{-q} N_{H}(\alpha, 0), \\
\lim _{\alpha \rightarrow \infty} \alpha^{-q} N(\alpha,-1)=0 .
\end{gathered}
$$

Proof. It follows from (1.14) and (3.5) that the functionals $\Delta_{q}^{( \pm)}$and $\delta_{q}^{( \pm)}$are the same for $X(1)$ and $Z(1)$; the same assertion is true for the operators $X(-1)$ and $Z(-1)$. Hence the necessary statements follow from (2.13) and (2.14). To obtain (3.8) we have to take into account (see $(1.13)$ ), that $Z(-1) \leq 0$.

Propositions 2.5 and 3.5 imply

Theorem 3.6. Let the condition (1.3) be fulfilled, and

$$
V(x)=V_{\tau}(x)(1+o(1)), \quad|x| \rightarrow \infty, 0<\tau<\frac{1}{3},
$$

where $V_{\tau}$ is the potential (2.20). Then we have (3.8) and the following asymptotic formula holds

$$
\lim _{\alpha \rightarrow \infty} \alpha^{-q} N(\alpha, 1)=2^{q+1} M_{\tau}, \quad \tau q=1
$$

where the coefficient $M_{\tau}$ is defined in (2.23).

Proof. For the potential $V=V_{\tau}$ the inclusion (3.5) is fulfilled (by (2.24) and also (1.3)). Therefore the formula (3.8) holds for the potential $V_{\tau}$, and the relations (3.6) and (3.7) by (2.24) transfer into (3.10). The same is true for the potential $V=\left(1-\chi_{R}\right) V_{\tau}, R>1$, since $\left(\chi_{R}-\chi_{1}\right) V_{\tau} \in L_{3 / 2}$, and the right hand sides in (3.6) and (3.7) do not change when we vary the potential $V$ with a term from the class $L_{3 / 2}\left(\mathbf{R}^{3}\right)$. By the same argument an arbitrary potential (3.9) can be substituted by another one which is equal to zero in the ball $\{|x|<R\}$. It is clear now that the asymptotics (3.10) are true for any potential $V \in L_{3}$ satisfying (3.9). 
§3.4. Let us now consider the case when (3.5) is fulfilled by $q=3$, or, which is the same when

$$
N_{H}(\alpha, 0)=O\left(\alpha^{3}\right)
$$

In this case both terms of the sum (1.19) give contributions of the same order. With some additional conditions we succeed in proving that their asymptotic contributions are added. The point is that the main contribution of the operator $Y$ to the spectrum is given by large momenta, but the contribution of the operator $Z( \pm 1)$ is given by small momenta. (The precise meaning of this statement is given in Lemmas 4.1 and 4.2.) Therefore the contributions of both terms enter into the asymptotic formula additively. It is convenient to express the necessary supplementary condition in terms of the Fourier transform $\widehat{W}=\Phi W, W=V^{1 / 2}$ :

$$
\int_{|\xi|>\varepsilon}|\widehat{W}(\xi)|^{2} d \xi<\infty, \quad \varepsilon>0
$$

Theorem 3.7. Let the conditions (1.3) and (3.11) be fulfilled and let us assume (3.12) for some $\varepsilon>0$. Then

$$
\begin{gathered}
\limsup _{\alpha \rightarrow \infty} \alpha^{-3} N(\alpha, 1)=J+16 \limsup _{\alpha \rightarrow \infty} \alpha^{-3} N_{H}(\alpha, 0), \\
\liminf _{\alpha \rightarrow \infty} \alpha^{-3} N(\alpha, 1)=J+16 \liminf _{\alpha \rightarrow \infty} \alpha^{-3} N_{H}(\alpha, 0), \\
\lim _{\alpha \rightarrow \infty} \alpha^{-3} N(\alpha,-1)=J .
\end{gathered}
$$

From Theorem 3.7 it is easy to deduce the analogy of Theorem 3.6.

Theorem 3.8. Let (1.3) be fulfilled and

$$
V(x)=\tilde{V}(x)(1+o(1)), \quad|x| \rightarrow \infty,
$$

where*

$$
\tilde{V}(x)=\left(1-\chi_{2}(x)\right) \frac{\Psi(\theta)}{|x|^{2}(\ln |x|)^{1 / 3}}, \quad \Psi \in L_{3}\left(\mathbf{S}^{2}\right)
$$

* The potential $V_{1 / 3}$ from (2.20) would not be convenient now, since it is not from $L_{3}\left(\mathbf{R}^{3}\right)$ because of the singularities at $|x|=1$. 
Then we have (3.15) and the following asymptotics hold

$$
\lim _{\alpha \rightarrow \infty} \alpha^{-3} N(\alpha, 1)=J+16 M_{1 / 3} .
$$

Proof. As in the proof of Theorem 3.6, it is enough to consider the case when in (3.16) $V=\widetilde{V}$. The following estimate is true

$$
\|X( \pm 1)\|_{\Sigma_{3}} \leq C|| \Psi \|_{L_{3}\left(\mathbf{S}^{2}\right)} .
$$

Indeed, from (1.19) it is enough to show similar estimates for the operators $Y$ and $Z( \pm 1)$. The estimate for the operator $Y$ is a simple corollary of the representation (1.11) and (2.6) when $d=3$ and $p=6$. The estimate for the operators $Z( \pm 1)$ by (1.12) follows from (2.18) with $q=3$ (we have to calculate the right hand side in (2.18) with $V=\widetilde{V}$ and $\varphi=|x|^{-2}$ ).

By (3.17) it is sufficient to calculate the spectral asymptotics for the operators $X( \pm 1)$ (and therefore the asymptotics of $N(\alpha, \pm 1)$ ) on some $L_{3}$-dense set in the cone set of functions $\Psi$ such that $\left\{\Psi \in L_{3}\left(\mathbf{S}^{2}\right): \Psi(\theta) \geq 0\right\}$. In particular, we may regard that

$$
\Psi \in C^{\infty}\left(\mathbf{S}^{2}\right), \quad \Psi(\theta)>0 .
$$

Then the condition (3.12) is fulfilled for any $\varepsilon>0$. It remains to apply Theorem 3.7 and Proposition 2.5 with $q=3$.

$\S 3.5$. The value

$$
N(\alpha, \Lambda):=N(\alpha, 1)-N(\alpha,-1)
$$

is equal to the number of all eigenvalues of the operator $\mathcal{D}(\alpha)$ inside the gap $\Lambda=(-1,1)$. Under conditions of Theorems 3.3 and 3.4, for the function (3.18) we obtain only the estimate $N(\alpha, \Lambda)=o\left(\alpha^{3}\right)$. On the contrary, under the conditions of Subsections 3 and 4 we obtain for $N(\alpha, \Lambda)$ more interesting asymptotic information.

\section{$\S 4$ The proof of Theorem 3.7}

§4.1. First of all let us make sure that the main contribution to the spectrum of the operator $Y$ is given by large momenta, and the main contribution to the spectrum of the operator $Z( \pm 1)$ is obtained from small momenta.

Let us settle the notations. In what follows below we denote by $\chi_{R}$ the characteristic function of the ball $\{|x|<R\} ; \widetilde{\chi}_{R}=1-\chi_{R}$. Set $(\boldsymbol{\gamma} \cdot \boldsymbol{D})|\boldsymbol{D}|^{-1}=S$. Using the notation (2.5) we represent the operator $Y$ as

$$
Y=Q_{1} S Q_{1}^{*} \text {. }
$$


Lemma 4.1. Let the condition (1.3) be satisfied and

$$
\widetilde{Y}_{R}:=Q_{1} \widetilde{\chi}_{R}(D) S \widetilde{\chi}_{R}(D) Q_{1}^{*}, \quad R>0 .
$$

Then

$$
Y-\widetilde{Y}_{R} \in \Sigma_{3}^{0} .
$$

Proof. From (2.6) we have $Q_{1} \in \Sigma_{6}$. Therefore to prove (4.1) it is sufficient to obtain the inclusion

$$
Q_{1} \chi_{R}(D) \in \Sigma_{6}^{0} .
$$

The last one follows directly from Proposition 2.1(b), since

$$
\left(Q_{1} \chi_{R}(D) v\right)(x)=(2 \pi)^{-3 / 2} W(x) \int_{|\xi| \leq R}|\xi|^{-1 / 2} e^{i x \xi} v(\xi) d \xi .
$$

Let us rewrite the operator $Z( \pm 1)$ in the form

$$
Z( \pm 1)=Q_{2}\left(\gamma_{0} \pm \mathbf{1}\right) Q_{2}^{*}
$$

and denote

$$
Z_{r}( \pm 1):=Q_{2} \chi_{r}(D)\left(\gamma_{0} \pm \mathbf{1}\right) \chi_{r}(D) Q_{2}^{*}, \quad r>0 .
$$

Lemma 4.2. Under conditions (1.3) and (3.11) we obtain

$$
Z( \pm 1)-Z_{r}( \pm 1) \in \Sigma_{3}^{0} .
$$

Proof. The equality (2.11) implies that the condition (3.11) is obviously equivalent to the inclusion $Q_{2} \in \Sigma_{6}$. Thus to obtain (4.2) it is sufficient to show that

$$
Q_{2} \widetilde{\chi}_{r}(D) \in \Sigma_{6}^{0} \text {. }
$$

The relation (4.3) can be established by applying the method of real interpolation to the integral operator

$$
\left(Q_{2} \widetilde{\chi}(D) v\right)(x)=(2 \pi)^{-3 / 2} W(x) \int_{|\xi| \geq r}|\xi|^{-1} e^{i x \xi} v(\xi) d \xi .
$$

Indeed, it is obvious that

$$
W \in L_{\infty}\left(\mathbf{R}^{3}\right) \quad \Rightarrow \quad Q_{2} \widetilde{\chi}_{r}(D) \in \mathcal{B}\left(L_{2}\right),
$$

and by Proposition 2.1

$$
W \in L_{3}\left(\mathbf{R}^{3}\right) \quad \Rightarrow \quad Q_{2} \widetilde{\chi}_{r}(D) \in \Sigma_{3}\left(L_{2}\right) .
$$

Interpolating the two last implications we find that

$$
W \in L_{6}\left(\mathbf{R}^{3}\right) \quad \Rightarrow \quad Q_{2} \tilde{\chi}_{r}(D) \in \mathfrak{S}_{6}\left(L_{2}\right) \subset \Sigma_{6}^{0} .
$$

In the formulation of the next lemma we avoid the obvious generalizations. 
Lemma 4.3. Let the condition (3.12) be fulfilled. Then

$$
P:=\widetilde{\chi}_{r+\varepsilon}(D) Q_{2} \chi_{r}(D) \in \mathfrak{S}_{2}
$$

Proof. Obviously the kernel of the operator $P$ is equal to

$$
(2 \pi)^{-3 / 2} \widetilde{\chi}_{r+\varepsilon}(\xi) \widehat{W}(\eta-\xi)|\eta|^{-1} \chi_{r}(\eta)
$$

Therefore

$$
\begin{aligned}
(2 \pi)^{3}\|P\|_{\mathfrak{S}_{2}}=\int_{|\eta| \leq r}|\eta|^{-2} d \eta \int_{|\xi| \geq r+\varepsilon} & |\widehat{W}(\eta-\xi)|^{2} d \xi \\
& \leq \int_{|\eta| \leq r}|\eta|^{-2} d \eta \int_{|\xi| \geq \varepsilon}|\widehat{W}(\xi)|^{2} d \xi<\infty
\end{aligned}
$$

§4.2. We now need the approximations of the operators $\widetilde{Y}_{R}$ and $Z_{r}( \pm 1)$.

Lemma 4.4. Let the condition (1.3) be satisfied. Let

$$
Y_{R}:=\widetilde{\chi}_{R}(D) Q_{1} S Q_{1}^{*} \widetilde{\chi}_{R}(D), \quad R>0
$$

Then

$$
\tilde{Y}_{R}-Y_{R} \in \Sigma_{3}^{0}
$$

Proof. Let us use the following representation

$$
\widetilde{Y}_{R}-Y_{R}=\left[W, \widetilde{\chi}_{R}(D)\right] S \widetilde{\chi}_{R}(D) Q_{2}^{*}+\widetilde{\chi}_{R}(D) Q_{2} S\left[\widetilde{\chi}_{R}(D), W\right]=: Y_{R, 1}+Y_{R, 2},
$$

where, as usual, the brackets [.,.] denote the commutator. Let us estimate for example the operator $Y_{R, 1}$. Representing the commutator explicitly we have

$$
Y_{R, 1}=Q_{1} \widetilde{\chi}_{R}(D) S \widetilde{\chi}_{R}(D) Q_{1}^{*}-\widetilde{\chi}_{R}(D) Q_{1} S \widetilde{\chi}_{R}(D) Q_{1}^{*}
$$

From (2.6) we find

$$
\left\|Q_{1}\right\|_{\Sigma_{6}} \leq C\|W\|_{L_{6}}
$$

which implies

$$
\left\|Y_{R, 1}\right\|_{\Sigma_{3}} \leq C|| W \|_{L_{6}}^{2} .
$$


The estimate (4.5) allows us to consider $W$ from any dense subset of $L_{6}\left(\mathbf{R}^{3}\right)$. Therefore we can assume that

$$
W \in C_{0}^{\infty}\left(\mathbf{R}^{3}\right)
$$

Then

$$
\begin{aligned}
Y_{R, 1} & =\left[\chi_{R}(D), W\right] S \tilde{\chi}_{R}(D) Q_{2}^{*} \\
& =\chi_{R}(D) W S \tilde{\chi}_{R}(D) Q_{2}^{*}-W \chi_{R}(D) S \widetilde{\chi}_{R}(D) Q_{2}^{*} .
\end{aligned}
$$

It is clear that under condition (4.6) $\chi_{R}(D) W \in \mathfrak{S}_{2}$ and $Q_{2} \in \Sigma_{3}$. Therefore both terms on the right hand side of (4.7) belong to the class $\Sigma_{3}^{0}$. Using the estimate (4.5) we see that the inclusion $Y_{R, 1} \in \Sigma_{3}^{0}$ can be extended to an arbitrary $W \in L_{6}$. Similarly one can prove that $Y_{R, 2} \in \Sigma_{3}^{0}$.

Denote now

$$
Z_{r, \varrho}( \pm 1):=\chi_{\varrho}(D) Z_{r}( \pm 1) \chi_{\varrho}(D), \quad \varrho>0 .
$$

Lemma 4.5. Let the conditions (3.11) and (3.12) be fulfilled, and let $\varrho=r+\varepsilon$. Then

$$
Z_{r}( \pm 1)-Z_{r, \varrho}( \pm 1) \in \Sigma_{3}^{0}
$$

Proof. The operator in (4.8) can be written as the following sum of three terms

$$
\begin{gathered}
\tilde{\chi}_{\varrho}(D) Z_{r}( \pm 1) \tilde{\chi}_{\varrho}(D)+\tilde{\chi}_{\varrho}(D) Z_{r}( \pm 1) \chi_{\varrho}(D)+\chi_{\varrho}(D) Z_{r}( \pm 1) \tilde{\chi}_{\varrho}(D) \\
=: \Omega_{1}+\Omega_{2}+\Omega_{2}^{*} .
\end{gathered}
$$

Using the notation introduced in (4.4), we write $\Omega_{1}$ in the form

$$
\Omega_{1}=P\left(\gamma_{0} \pm 1\right) P^{*} \text {. }
$$

From Lemma 4.3 we obtain that $\Omega_{1} \in \mathfrak{S}_{1} \subset \Sigma_{3}^{0}$. We have

$$
\Omega_{2}=P\left(\gamma_{0} \pm \mathbf{1}\right) \chi_{r}(D) Q_{2}^{*} \chi_{\varrho}(D) .
$$

Here $P \in \mathfrak{S}_{2}$, and the other terms are bounded (moreover, (3.11) implies that $Q_{2} \in \Sigma_{6}$ ). Therefore certainly $\Omega_{2} \in \Sigma_{3}^{0}$. This implies that the operator (4.9) belongs to $\Sigma_{3}^{0}$.

$\S 4.3$. The proof of Theorem 3.7. Using (1.7), (2.11) and also (2.13), (2.14) we rewrite the asymptotic formulae (3.13), (3.14) and (3.15) as follows

$$
\Delta_{3}^{(+)}(X(1))=J+16 \Delta_{3}\left(Z_{H}\right),
$$




$$
\begin{gathered}
\delta_{3}^{(+)}(X(1))=J+16 \delta_{3}\left(Z_{H}\right), \\
\Delta_{3}^{(+)}(X(-1))=\delta_{3}^{(+)}(X(-1))=J .
\end{gathered}
$$

Let us agree to denote the terms from the class $\Sigma_{3}^{0}$ by dots. Using the lemmas of this section we have

$$
X( \pm 1)=Y+Z( \pm 1)=\widetilde{Y}_{R}+Z_{r}( \pm 1)+\ldots=Y_{R}+Z_{r, \varrho}( \pm 1)+\ldots .
$$

Let $R>\varrho$. Then the operators $Y_{R}$ and $Z_{r, \varrho}( \pm 1)$ are mutually orthogonal, and therefore

$$
\Delta_{3}^{(+)}(X( \pm 1))=\Delta_{3}^{(+)}\left(Y_{R}\right)+\Delta_{3}^{(+)}\left(Z_{r, \varrho}( \pm 1)\right) .
$$

We now have

$$
Y_{R}-Y \in \Sigma_{3}^{0}, \quad Z_{r, \varrho}( \pm 1)-Z( \pm 1) \in \Sigma_{3}^{0}
$$

and hence

$$
\Delta_{3}^{(+)}(X( \pm 1))=\Delta_{3}^{(+)}(Y)+\Delta_{3}^{(+)}(Z( \pm 1))
$$

Similarly,

$$
\delta_{3}^{(+)}(X( \pm 1))=\delta_{3}^{(+)}(Y)+\delta_{3}^{(+)}(Z( \pm 1)) .
$$

It is clear now that by $(1.14),(2.13),(2.14)$ the equalities $(4.13)_{+}$and $(4.14)_{+}$lead to $(4.10)$ and(4.11). In just the same way we have that (4.13) ${ }_{-}$and $(4.14)_{-}$are equivalent to (4.12), since $Z(-1) \leq 0$.

\section{References}

[BL] BerGH, J. and LÖFSTRÖM, J., Interpolation Spaces. An Introduction., Grundlehren der mathematischen Wissenschaften 223, Springer-Verlag, BerlinHeidelberg-New York, 1976.

[B1] BiRman, M. SH., Discrete spectrum in the gaps of a continuous one for perturbations with large coupling constant, Adv. Soviet Math. 7 (1991), 57-73.

[B2] BIRMAN, M. Sh., Discrete spectrum in a gaps of a perturbed periodic Schrödinger operator. I. Regular perturbations, to appear in Adv. Soviet Math.

[BKS] Birman, M. Sh., Karadzhov, G. E. and SolomyaK, M. Z., Boundedness conditions and spectrum estimates for the operators $b(X) a(D)$ and their analogs, Adv. Soviet Math. 7 (1991), 85-106.

[BS1] Birman, M. Sh. and SolomyaK, M. Z., Asymptotic behavior of the spectrum of weakly polar integral operators, Izv. Akad. Nauk. SSSR Ser. Mat. 34 (1970), 1142-1158 (Russian). English transl.: Math. USSR-Izv. 4 (1970), 1151-1168. 
[BS2] BiRman, M. SH. and SolomyaK, M. Z., Spectral asymptotics of pseudodifferential operators with anisotropic homogeneous symbols. I, Vestnik Leningrad. Univ. Mat. Mekh. Astronom. 13 (1977), 13-21. II, Vestnik Leningrad. Univ. Mat. Mekh. Astronom. 13 (1979), 5-10 (Russian).

[BS3] Birman, M. Sh. and Solomyak, M. Z., Spectral Theory of Selfadjoint Operators in Hilbert Space, D. Reidel Publ. Comp., Dordrecht-Boston, 1987.

[BS4] Birman, M. SH. and SolomyaK, M. Z., Estimates for the number of negative eigenvalues of the Schrödinger operator and its generalizations, Adv. Soviet Math. 7 (1991), 1-55.

[BS5] Birman, M. Sh. and SolomyaK, M. Z., Schrödinger Operator. Estimates for number of bound states as function-theoretical problem, Amer. Math. Soc. Transl. (2) 150 (1992), 1-54.

[C] CWIKEL, M., Weak type estimates for singular values and the number of bound states of Schrödinger operators, Ann. of Math. 106 (1977), 93-100.

[K] KLaus, M., On the point spectrum of Dirac operators, Helv. Phys. Acta 53 (1980), 453-462.

[L] LAPTEV, A., Asymptotics of the negative discrete spectrum of a class of Schrödinger operators with large coupling constant, Proc. Amer. Math. Soc. (2) 119 (1993), 481-488.

[T] Thaller, B., The Dirac Equation, Texts and Monographs in Physics, SpringerVerlag, Berlin-Heidelberg-New York, 1992.

[Y] YafaEv, D., Mathematical Scattering Theory, Transl. Math. Monographs 105, Amer. Math. Soc., Providence, R.I., 1992.

Mikhail Sh. Birman

St. Petersburg University

Department of Physics

Ulyanov str. 1

198904 St. Petersburg

Russia

Ari Laptev

Department of Mathematics

Royal Institute of Technology

S-100 44 Stockholm

Sweden

email: laptev@math.kth.se 